\title{
Evidence for Quasiparticle Decay in Photoemission from Underdoped Cuprates
}

\author{
R. B. Laughlin \\ Department of Physics, Stanford University, Stanford, California 94305 USA
}

(Received)

\begin{abstract}
I argue that the "gap" recently observed at the Brillouin zone face of cuprate superconductors in photoemission by Marshall et al [Phys. Rev. Lett. 76, 4841 (1996)] and Ding et al [Nature 382, 54 (1996)] is evidence for the decay of the injected hole into a spinon-holon pair.
\end{abstract}

PACS numbers: 74.20.Mn, 74.72.-h, 79.60.-i

One of the most interesting developments in cuprate superconfuctivity is the the recent observation by Marshall et all and Ding et ale of a pseudogap in the electron spectral function near the Brillouin zone face that persists above the superconducting transition temperature and grows in magnitude as doping is reduced. This feature, which is also seen in optical conductivity 3 and is almost certainly the "spin gap" effect seen in magnetic resonance 1 , has the momentum dependence expected of a simple d-wave superconductor but a size, doping dependence, and breadth that do not, particularly at low dopings.

The purpose of this paper is to propose that these experiments may constitute long-sought evidence that spinons and holons, the soliton-like particles known from studies of 1-dimensional antiferromagnets $\bar{E}$, actually exist in these materials. The reason is that there is no other simple explanation of the experiments that is not also contrived. For example, the evolution of the feature out of the d-wave gap with underdoping has led to speculation that it is the dissociation of a "pre-formed" Cooper pair, this being a specific realization of the quite sensible ideas of Kivelson and Emeryb. However, the attractive force required to accomplish such pairing would be outrageously large, no such effect has ever been observed in a conventional metal, and the effect persists to extreme underdoping where the material is an insulator. Similarly, the practice of modeling the system as a spin density wave does not work in situations lacking longrange order, requires delicate adjustments of the distantneighbor hopping integrals to account for the observed isotropy of the quasiparticle dispersion relation, and does not account at all for the enormous width of the quasiparticle peak at the zone face. The last two remarks appty broadly to existing work on the $t-t^{\prime}-J$ model as wellt. The discussion I shall present deliberately avoids sophisticated mathematics and argues directly from the experiments shown in Figs. 1 and 2 and the consistency of these with Eqs. (1) - (4). A formalism-free approach is essential because the objective is not to make a theory of high-Tc superconductivity - a delicate question of symmetry breaking - to promote a model or to report calculations, but rather to establish that spinons and holons are real.

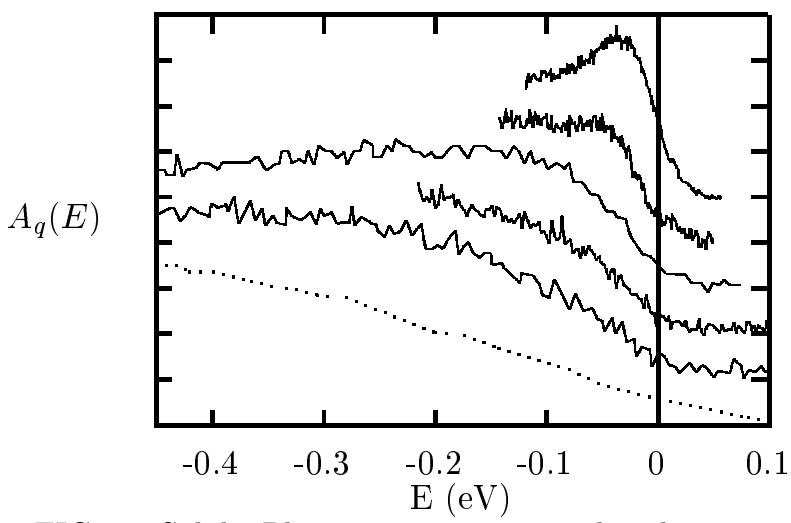

FIG. 1. Solid: Photoemission energy distribution curves near the X-point of BSCCO at various dopings as described in the text. Dashed: $X^{\prime}$ curve of the magnetic insulator $\mathrm{Sr}_{2} \mathrm{CuO}_{2} \mathrm{Cl}_{2}$ taken from Fig. 2 with the zero of energy shifted by $0.7 \mathrm{eV}$ to account for the chemical potential difference.

The spinon and holon I have proposed to be present in the cuprates have the dispersion relations

$$
\begin{gathered}
E_{k}^{\text {spinon }}=1.6 J \sqrt{\cos ^{2}\left(k_{x} b\right)+\cos ^{2}\left(k_{y} b\right)}, \\
E_{k}^{\text {holon }}= \pm 2 t \sqrt{\cos ^{2}\left(k_{x} b\right)+\cos ^{2}\left(k_{y} b\right)},
\end{gathered}
$$

where $\mathrm{t}=0.5 \mathrm{eV}$ and $\mathrm{J}=0.125 \mathrm{eV}$ are the bandwidth and magnetic exchange parameters of a magnetic Hamiltonian such as the t-J model and $b=4$ Ai the bond length. I wish to be somewhat vague about the specifics of the Hamiltonian because it is not known whether any such model describes the cuprates in detail. Fortunately Eqs. (1) and (2), unlike questions of order, are insensitive to subtleties. The values of the parameters are important. $t$ is a tight-binding fit to the bare HartreeFock-Slater band structure 3 and is a number characterizing charge transport. His a Heisenberg fit to the 2magnon raman scattering 9 and neutron scattering 10 experiments performed on the insulator and is a number characterizing the magnetism. Both parameters should be considered known and not adjusted later to fit other experiments.

The "new" development motivating this paper is the discovery of the spin gap in underdoped superconductors. 
I have recently written a series of papers arguing that spinons and holons may be seen indirectly in numerical

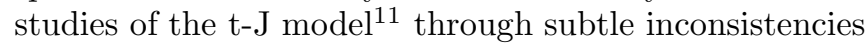
of sum rules as $\mathrm{t}$ and $\mathrm{J}$ are varied 2 . A more direct observation has not been possible - until now - because the violent attraction of these particles for each other so distorts the commonly calculated spectra that their shape becomes mostly a measure of the interaction 12 . These experiments, however, imply that the interaction is substantially weaker in the cuprates than it is in the t-J model at low doping. There are many potential causes of this - doping, elevation of the temperature, or modification to the Hamiltonian, for example - and distinguishing among these is quite beyond our means at present. So we must defer the question of cause for now, or more precisely restrict ourselves to versions of the question that have experimental answers. For example, I currently favor the theory that the finite temperature required to prevent the sample from charging is the cause of this weakness in the insulators. The only reasonable test of this is to repeat the experiment cold and see if the t$\mathrm{J}$ results materialize. But regardless of the cause, the weakened interaction is the key difference between the experiments and the t-J studies at low doping.

Let me begin by making a connection between the insulators and metals. In the inset of Fig. 1 I show a compilation of energy distribution curves taken by two different experimental groups near the X-point of BSCCO for various dopings. The samples were made in different laboratories and have slightly different stoichoimetries. The short curves are, top to bottom, the $T_{c}=87 \mathrm{~K}$, $83 \mathrm{~K}$, and $10 \mathrm{~K}$ data taken from Fig. 1 of Ding et all, which correspond to samples of $\mathrm{Bi}_{2} \mathrm{Sr}_{2} \mathrm{CaCu}_{2} \mathrm{O}_{8+\delta}$ measured at the $(\pi, 0)-(\pi, \pi)$ fermi surface crossing. The long curves are, top to bottom the Tc $=65 \mathrm{~K}(\pi, 0)$ curve of Fig. 2 of Marshall et alt, which corresponds to a $\mathrm{Bi}_{2} \mathrm{Sr}_{2} \mathrm{Ca}_{1-x} \mathrm{D}_{4} \mathrm{Cu}_{2} \mathrm{O}_{8+\delta}$ sample at $10 \%$ Dy doping, and unpublished 13 data for an insulating sample of this material with $35 \%$ Dy doping. When they are plotted on the same graph in this way it becomes obvious that the data on the underdoped superconductors interpolate between the behavior found at optimal doping, which is roughly consistent with conventionalmetal physics, and the behavior reported by Wells et a 14 for the magnetic insulator $\mathrm{Sr}_{2} \mathrm{CuO}_{2} \mathrm{Cl}_{2}$ reproduced in Fig. 2. This fact has two major implications. The first is that the strange behavior of the insulator near the X-point is not an artifact of the particular material, but is generic to the cuprates and therefore worth understanding. This was not clear when it was first discovered. The second is that it is the same effect as the spin gap. This is an extremely strong statement but it is clearly true, for otherwise we would need to invent two independent mechanisms for producing "d-wave" gapping in this problem and explain why one of them continuously evolved into the other with doping. The study of the insulator and the study of the spin gap are the same thing.
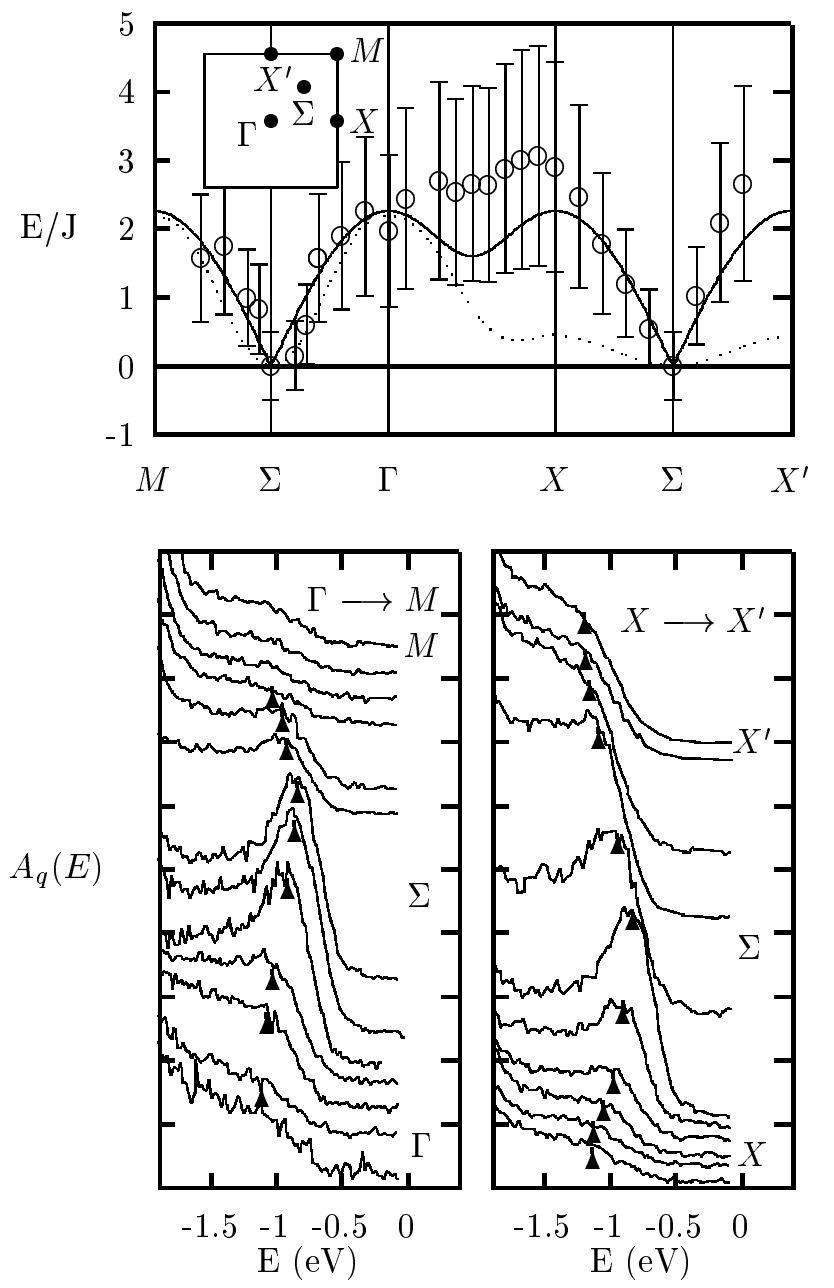

FIG. 2. Top: Comparison of "quasiparticle" dispersion relation found in insulating $\mathrm{Sr}_{2} \mathrm{CuO}_{2} \mathrm{Cl}_{2}$ by Wells et al with the prediction of Eq. (1). The error bars represent my estimate of the width of the peak and not the accuracy of the measurement. The dashed curve is the dispersion relation found by numerical t-J studies. Bottom: Photoemission energy distribution curves from which this dispersion relation was inferred. The arrows correspond to the open circles in the top panel.

Let us now consider the question of quasiparticle integrity. It may be seen in Fig. 2 that the energy distribution curves of $\mathrm{Sr}_{2} \mathrm{CuO}_{2} \mathrm{Cl}_{2}$ show a peak that disperses with momentum, has its lowest energy at $\Sigma$, and broadens substantially away from this minimum. Along the line $\Gamma \rightarrow M$ the data are essentially indistinguishable from those of the superconductors at any doping, not only in shape but in scale, so nothing is lost by plotting only the insulator data. It may also be seen that the spectra at the extremal points $\Gamma, M$, and $X$ are so broad that they are more properly characterized as a continuum with the hint of a knee or edge about $0.2 \mathrm{eV}$ above the fermi energy. This edge was interpreted by Wells et al 14 as the quasiparticle peak at this momentum and plotted as a point in their quasiparticle dispersion relation, also reproduced in Fig. 2. While this is a reasonable thing to do if the quasiparticle is assumed to exist, it is very 
unreasonable otherwise, for the ostensible lifetime broadening at these momenta is greater than the entire dispersion across the zone. We know this broadening to be intrinsic and not caused by surface disorder because the same sample shows a well-defined peak near $\Sigma$ and strong angle dependence of this peak. Also, all the cuprates show such broadening at $\Gamma$ and $M$. So these data actually imply that the quasiparticle has no integrity at these momenta at all, and does not, in fact, exist.

Let us now consider the energy scale of the quasiparticle. It is now well established that the quasiparticle bandwidth in all the cuprates is about $0.3 \mathrm{eV}$ regardless of crystal structure or doping level, a rather astonishing fact in light of their different transport and optical properties. It may be seen in Fig. 2, for example, that the quasiparticle energy at $\Gamma, M$, and $X$, insofar as it is defined, is 0.3 $\mathrm{eV}$ higher than that at $\Sigma$. This energy scale is an important clue to the nature of the microscopic physics because it is so peculiar. Conventional metals and semiconductors have bandwidths ten times larger than this because their energy scale is set by the matrix element for electrons to hop between adjacent sites - typically 1 or $2 \mathrm{eV}$. This is why the cuprate bandwidth is 2-3 times smaller than that predicted by conyentional Hartree-Fock-Slater band structure calculations 8 and well outside their expected error bar. Nor is it reasonable to ascribe this energy to phonons. The cuprates are ionic and thus have large electron-phonon couplings, but not larger than those in alkalai halides, where the effect of phonons is either to enhance the band mass slightly, as occurs in the conduction band, or to enhance it by many orders of magnitude through the small polaron effect, as occurs in the valence band. Indeed the only energy in the problem the right size to account naturally for this bandwidth is the magnetic exchange parameter J. One of the strangest and most consistent findings of the numerical work on the t-J model 15 at low doping has been that the quasiparticle bandwidth is $2.2 \mathrm{~J}$ regardless of the value of t12. Since the bandwidth does not require high resolution to compute and is known to be relatively insensitive to other parameters such as $t^{\prime}$, it must be considered a firm prediction of these calculations that the bandwidth should be about $0.3 \mathrm{eV}$. Thus the agreement between the prediction of the model and experiment suggests that the t-J model has some relevance to the problem and that the quasiparticle bandwidth is set by $\mathrm{J}$.

Let us finally consider the question of isotropy. It may be seen from Fig. 2 that the energy scale of the quasiparticle at $\Gamma, M$, and $X$ is the same, and that the dispersion near $\Sigma$, where the peak is sharpest, is isotropic. This isotropy does not agree with the t-J studies at low doping, which match experiment in the $\Gamma \rightarrow M$ direction but show no dispersion at all $X \rightarrow X^{\prime}$ direction. This disparity has led a number of theorists to add other parameters, typically a second-neighbor hopping integral $t^{\prime}$, to the Hamiltonian and adjust its value to make the quasiparticle dispersion in an approximate calculation match experiment everywhere. In addition to failing to account for the quasiparticle width, this line of reasoning has the obvious flaw of ascribing the isotropy to a coincidence of the parameters $\mathrm{J}$ and $t^{\prime}$, notwithstanding the strangeness of the energy scale. While such a coincidence is conceivable, it is far more reasonable to conclude that the dispersion in the $X \rightarrow X^{\prime}$ direction is regulated by the same parameter regulating the $\Gamma \rightarrow M$ dispersion, namely $\mathrm{J}$, and that the failure of the t-J calculations to find this effect is a subtle problem related to their failure to find the correct quasiparticle width at $\mathrm{X}$.

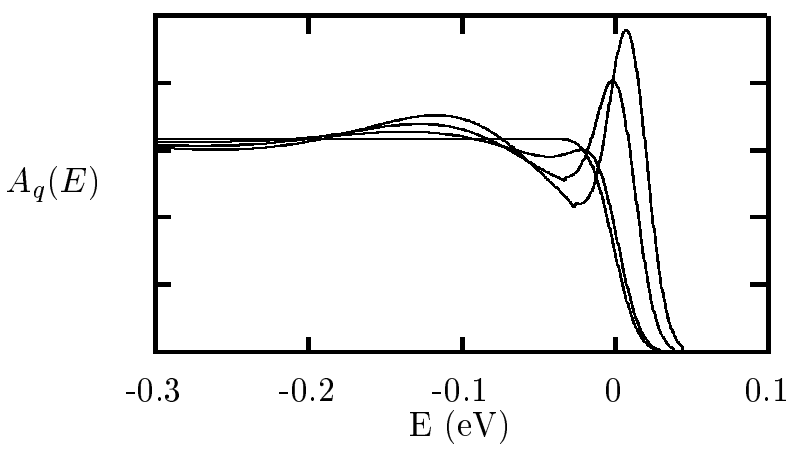

FIG. 3. Theoretical spectral function defined by Eq. (3) for $V_{0}=0.00 \mathrm{eV}, 0.01 \mathrm{eV}, 0.02 \mathrm{eV}$, and $0.03 \mathrm{eV}$. The spectrum has been convolved with a gaussian of width $0.012 \mathrm{eV}$.

I now turn to my interpretation of these experiments, namely that the injected hole is decaying into a spinonholon pair. The disparity between $\mathrm{t}$ and $\mathrm{J}$ in our problem is such that decay is expected to result in a continuum several electron volts wide and an edge tracing out the spinon dispersion relation, the minimum-energy decay being when the spinon carries away all the momentum. If there is in addition a weak attractive force between the spinon and holon, structure develops at this edge. In Fig.3 I plot the spectrum

$$
A(E)=\sum_{n}\left|\psi_{n}(0)\right|^{2} \delta\left(E+E_{n}\right)
$$

where

$$
\begin{gathered}
\mathcal{H} \psi_{n}(r)=E_{n} \psi_{n}(r) \\
\mathcal{H}=-\frac{\hbar^{2}}{2 m^{*}} \nabla^{2}-V_{0} \theta\left(r_{0}-r\right),
\end{gathered}
$$

which is a model 2-body Greens' function matrix element for the two particles to coincide in space in the limit that the spinon bandwidth is zero. The parameter $m^{*}=\hbar^{2} /\left(\sqrt{2} t b^{2}\right)$ is the holon band effective mass computed from Eq. (2) or 0.7 electron masses, the square-well radius is $r_{0}=6 b$ or $24 \AA$, and the depth is $0.0 \mathrm{eV} \leq V_{0} \leq 0.03 \mathrm{eV}$. The "quasiparticle" peak in this spectrum is a bound state of the spinon and holon which is small because the wavefunction in question is physically large. The features in the spectrum at higher energy are scattering resonances; note that these occur at 
an energy scale 10 times larger than the size of $V_{0}$, which is itself of order the superconducting $T_{c}$. In Fig. $2 \mathrm{I}$ compare the "flasiparticle" dispersion relation inferred by Wells et a14 with the spinon dispersion relation of Eq. (1). The agreement between the two is obviously excellent, including the overall energy scale, which is not adjustable, the equivalence of the energies at $\Gamma, M$, and $X$, and the isotropy near $\Sigma$. Thus except for the peaking of the spectrum near $\Sigma$, which I ascribe to dependence of the attractive force on the center-of-mass momentum, this experiment, and the spin gap experiments like it, are plausibly understood as the broad continuum expected from quasiparticle decay with a threshold at the energy of the bare spinon.

As Eqs. (1) and (2) are essential to the argument, let me now explain their origin. These are the dispersion relations for the spinon and holon found by a number of us in the early days of high- $T_{c}$. They are alternately described in papers of that time as behavior of an electron in a d-wave superconductor 16 , the behavior of an electron in a magnetic field of flux $\pi$ per plaquette 17 , and the behavior of a doubled Dirac fermion on a square lattice 19 . It later came to be understood that none of these had its literal pysical meaning and all were mathematically equivalent18. The spinon and holon are actually fractional particles analogous to the charge carriers in the fractional quantum hall effect. The d-wave or flux "order" is simply the price one pays to describe such an object in conventional particle language. It is fictitious and disappears when the spinon and holon are written down as actual spin wavefunctions 20 . However, these are so complex that it is usually more convenient to work with an overcomplete basis and fictitious order. This is the underlying reason why descriptions of the antiferromagnet based on these particles are always gauge theories. The factors of $2 \mathrm{t}$ and $1.6 \mathrm{~J}$ are fits made by me in reconciling these equations with known properties of the t-J model. They have proper justifications within the context of the gauge theory, but this is less important than the fact that they were published before the experiments were performed.

Let me now show in a crude way how an attractive force between two such particles can account for the behavior found in the numerical work on the t-J model. A highly detailed explanation is undesirable here because it would amount to model building and add unnecessary complexity. We consider two particles moving on a square lattice and described by the Hamiltonian

$$
\mathcal{H}=\sum_{<j, k>} V_{j k}\left\{a_{j}^{\dagger} a_{k}+b_{j}^{\dagger} b_{k}\right\}-V_{0} \sum_{j} a_{j}^{\dagger} b_{j}^{\dagger} b_{j} a_{j}
$$

where $\langle j, k\rangle$ denotes the set of near-neighbor pairs, with each pair counted twice to maintain hermiticity, and $V_{j k}$ is taken to be $-V$ for the horizontal bonds in even rows and $+V$ for all remaining bonds. When the attractive interaction $V_{0}$ is turned off the particles are free and are described by the dispersion re- lation $E_{k}= \pm 2 V \sqrt{\cos ^{2}\left(k_{x}\right)+\cos ^{2}\left(k_{y}\right)}$, which has the functional form of Eqs. (1) and (2). When the attraction is very large, on the other hand, the particles form a bound state, the dispersion relation of which is $E_{k}=4 V^{2} / V_{0}\left[\cos \left(k_{x}\right)+\cos \left(k_{y}\right)\right]$, the functional form found in the t-J studies. The difference between these two behaviors is due to the bound state's being a whole particle rather than a fractional one, and thus blind to the fictitious magnetic field. The issues of dynamical scale of the bound state, the presence of two holon branches but only one spinon branch, and the detailed nature of the attractive force have all been discussed at length in previous papers12, but they are not conceptually important. The new and important observation is that the functional form of conventional bands is restored by gauge invariance when spinons and holons bind tightly, regardless of details.

The issue of whether spinons and holons are real is centrally important to high-Tc superconductivity. It must be squarely faced if there is to be a meaningful discussion of theories based on spin-charge separation.

I wish to thank Z.-X. Shen, and M. Norman for providing access to these data and for numerous helpful discussions. This work was supported by the NSF under grant No. DMR-9421888.

${ }^{1}$ D. S. Marshall et al, Phys. Rev. Lett. 76, 4841 (1996); A. G. Loeser et al, Science 273, 3235 (1996).

${ }^{2}$ H. Ding et al, Nature, 382, 51 (1996).

${ }^{3}$ C. C. Homes et al, Phys. Rev. Lett. 71, 1645 (1993); Z. Schlesinger et al, Physica C 235 - 240, 49 (1994).

${ }^{4}$ A. Millis and H. Monien, Phys. Rev. Lett. 70, 2810 (1993); ibid. 71, 210 (1993).

${ }^{5}$ P. W. Anderson, Science, 235, 1196 (1987).

${ }^{6}$ V. J. Emery and S. A. Kivelson, Nature 374, 434 (1995).

${ }^{7}$ A. Nazarenko et al, Phys. Rev. B 51, 8676 (1995).

${ }^{8}$ L. F. Mattheiss, Phys. Rev. Lett. 58, 1028 (1987).

${ }^{9}$ K. B. Lyons et al, Phys. Rev. B 37, 2353 (1988).

${ }^{10}$ S. M. Hayden et al, Phys. Rev. Lett. 67, 3622 (1991).

${ }^{11}$ E. Dagotto, Rev. Mod. Phys. 66, 763 (1994).

${ }^{12}$ R. B. Laughlin, J. Low Temp. Phys. 99, 443 (1995).

13 D. S. Marshall, PhD Thesis, Stanford University.

${ }^{14}$ B. O. Wells et al,Phys. Rev. Lett. 74, 964 (1995).

${ }^{15}$ D. Poilblanc et al, Phys. Rev. B 47, 14267 (1993).

${ }^{16}$ G. Baskeran, Z. Zou, and P. W. Anderson, Solid State Comm. 63, 973 (1987); Z. Zou, Phys. Rev. B 40, 2262 (1989).

${ }^{17}$ I. Affleck and J. B. Marston, Phys. Rev. B 37, 3774 (1988).

${ }^{18}$ G. Kotliar, Phys. Rev. B 37, 3664 (1988).

19 J. B. Kogut, Rev. Mod. Phys. 55, 775 (1983).

${ }^{20}$ Z. Zou and R. B. Laughlin, Phys. Rev. B 42, 4073 (1990); Z. Zou, J. L. Levy, and R. B. Laughlin, Phys. Rev. B 45, 993 (1992). 